\title{
Valley-dependent gauge fields for ultracold atoms in square optical superlattices
}

\author{
Dan-Wei Zhang, ${ }^{1}$ Chuan-Jia Shan, ${ }^{2,1}$ Feng Mei, ${ }^{3}$ Mou Yang, ${ }^{1}$ Rui-Qiang Wang, ${ }^{1}$ and Shi-Liang Zhu ${ }^{3,1, *}$ \\ ${ }^{1}$ Laboratory of Quantum Engineering and Quantum Materials, SPTE, South China Normal University, Guangzhou 510006, China \\ ${ }^{2}$ College of Physics and Electronic Science, Hubei Normal University, Huangshi 435002, China \\ ${ }^{3}$ National Laboratory of Solid State Microstructures and School of Physics, Nanjing University, Nanjing 210093, China
}

(Received 2 October 2013; published 21 January 2014)

\begin{abstract}
We propose an experimental scheme to realize the valley-dependent gauge fields for ultracold fermionic atoms trapped in a state-dependent square optical lattice. Our scheme relies on two sets of Raman laser beams to engineer the hopping between adjacent sites populated by two-component fermionic atoms. One set of Raman beams is used to realize a staggered $\pi$-flux lattice, where low-energy atoms near two inequivalent Dirac points should be described by the Dirac equation for spin- $\frac{1}{2}$ particles. Another set of laser beams with proper Rabi frequencies is added to further modulate the atomic hopping parameters. The hopping modulation will give rise to effective gauge potentials with opposite signs near the two valleys, mimicking the interesting strain-induced pseudogauge fields in graphene. The proposed valley-dependent gauge fields are tunable and provide an alternative route to realize an uncommon type of quantum Hall effects and atomic devices.
\end{abstract}

DOI: 10.1103/PhysRevA.89.015601

The low-energy effective theory of graphene describes relativistic Dirac fermions near the two inequivalent corners of the Brillouin zone, termed valleys [1]. Valley index plays an important role in the extraordinary electronic properties of graphene. Valley-dependent gauge fields, usually called pseudogauge fields to distinguish from the real valleyindependent electromagnetic field in unstrained graphene, have recently been studied extensively both theoretically [2-4] and experimentally $[5,6]$. It has been show that such gauge fields can be realized by modulating the electronic hopping with strains in a two-dimensional (2D) honeycomb lattice $[5,6]$. These findings open up an exciting area of mechanically engineering band structure of graphene [3], as well as realizing some exotic phenomena absent in other solid-state materials, such as new types of quantum Hall related effects $[4,7]$.

On the other hand, a growing class of Dirac materials with synthetic honeycomb structure have recently been proposed and explored [8], such as trapped cold atoms in optical lattices (OL) $[9,10]$, confined photons in photonic crystals [11,12], and molecular graphene [13]. Interestingly, the pseudomagnetic fields and related Landau levels have been experimentally demonstrated in photonic graphene [11] and molecular graphene [13] by designing a spatial texture of hopping parameters. In addition, the creation and manipulation of Dirac points with a Fermi gas in a honeycomb OL have been also reported recently [10]. A promising extension in this cold-atom system is to simulate the tunable valleydependent gauge fields and realize the related novel effects. For this purpose, a practical way is to modulate the atomic hopping parameters in a honeycomb OL by using the synthetic gauge potentials [14] or the laser-assisted tunneling (LAT) [15,16], following the schemes proposed in Refs. [17-19]. However, the LAT technique has not yet been demonstrated in honeycomb OLs, but in square optical (super)lattices [20-22]. Therefore, a natural question is whether one can simulate the valley-dependent gauge fields within current experimental technique in a square OL.

\footnotetext{
*slzhunju@163.com
}

PACS number(s): 67.85.-d, 03.75.Lm, 03.65.Pm

In this Brief Report, we propose a feasible scheme to realize the valley-dependent gauge fields for ultracold fermionic atoms trapped in a square optical superlattice. In our scheme, a state-dependent square OL populated by two-component atoms is considered and this lattice has a checkerboard configuration, which allows for engineering LAT in the two spatial directions. As the first step to simulate the valleydependent gauge fields, two Raman laser beams are employed to create a staggered $\pi$-flux lattice, which results in an effective relativistic Hamiltonian near the two inequivalent Dirac points. The second step is to further modulate the hopping amplitudes in the previous $\pi$-flux lattice by using another two or three Raman beams with proper Rabi frequencies. If the hopping modulation is smooth over the lattice spacing scale, it will give rise to effective gauge potentials with opposite signs near the two valleys, mimicking the interesting pseudogauge fields in strained graphene [2-7]. These synthetic gauge fields can be controlled by carefully designing the Rabi frequencies of the Raman laser beams in the second step. In addition, we briefly present some potential applications with these gauge fields, including the quantum valley Hall effect (QVHE) and atomic devices based on valley-dependent gauge fields. Although there have been a great deal of theoretical and experimental studies in producing artificial gauge fields for neutral atoms [14], none of them couple with the valley degree of freedom. So, our proposal can enlarge the community of gauge fields in cold-atom systems and provide a pathway towards realizing atomic valley-based devices.

Let us start by considering a 2D noninteracting twocomponent fermionic gas in a state-dependent square $\mathrm{OL}$ with a checkerboard structure, as shown in Fig. 1(a). Such state-dependent OLs have been experimentally created by superposing two linearly polarized laser beams with a relative polarized angle, where the separation and potential depth of the two sublattices $(A$ and $B$ ) can be well controlled by the angle and the laser intensity [23]. The fermionic atoms are commonly chosen as ${ }^{6} \mathrm{Li}$ or ${ }^{40} \mathrm{~K}$ in current experiments. For ${ }^{6} \mathrm{Li}$ atoms, the hyperfine levels for the two-component states can be $|A\rangle=\left|2^{2} S_{1 / 2}, \frac{3}{2},-\frac{1}{2}\right\rangle$ and $|B\rangle=\left|2^{2} S_{1 / 2}, \frac{3}{2}, \frac{3}{2}\right\rangle$, respectively. For ${ }^{40} \mathrm{~K}$ atoms, the hyperfine levels can be 

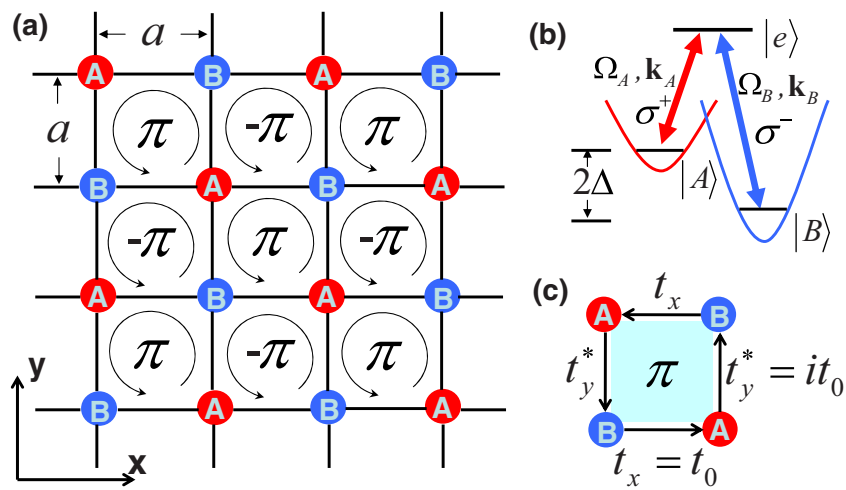

FIG. 1. (Color online) (a) Schematic two-dimensional statedependent OL with a checkerboard configuration and a staggered $\pi$ flux. (b) Sketch of laser-assisted tunneling between two nearestneighbor lattice cites. The atoms with internal ground states $|A\rangle$ and $|B\rangle$ are, respectively, trapped in sublattices $A$ and $B$, with a tunable onsite energy imbalance. They are coherently coupled to the excited state $|e\rangle$ through Raman laser beams with Rabi frequencies $\Omega_{A} e^{i \mathbf{k}_{A} \cdot \mathbf{r}}$ and $\Omega_{B} e^{i \mathbf{k}_{B} \cdot \mathbf{r}}$. (c) The hopping configuration of each plaquette for simulating a staggered $\pi$-flux lattice [16], with the hopping parameters along the $\hat{x}(\hat{y})$ axis as $t_{x}=t_{0}\left(t_{y}=-i t_{0}\right)$. For a particle hopping anticlockwise around a plaquette, the phase factor picked up along the path $(i, j)_{B} \rightarrow(i+1, j)_{A} \rightarrow(i+1, j+1)_{B} \rightarrow$ $(i, j+1)_{A} \rightarrow(i, j)_{B}$ is $e^{i \pi}$. For the adjacent plaquette, the phase factor is $e^{-i \pi}$.

$|A\rangle=\left|4^{2} S_{1 / 2}, \frac{7}{2},-\frac{1}{2}\right\rangle$ and $|B\rangle=\left|4^{2} S_{1 / 2}, \frac{7}{2}, \frac{3}{2}\right\rangle$. In this OL, the atoms must alter their internal states in order to tunnel between two nearest-neighbor lattice sites. This can be achieved by the so-called LAT method [15,16,20-22]. Two Raman laser beams with Rabi frequencies $\Omega_{A} e^{i \mathbf{k}_{A} \cdot \mathbf{r}}$ and $\Omega_{B} e^{i \mathbf{k}_{B} \cdot \mathbf{r}}$ are applied to couple the states $|A\rangle$ and $|B\rangle$ via an immediate excited state $|e\rangle$, as shown in Fig. 1(b). The excited states for ${ }^{6} \mathrm{Li}$ and ${ }^{40} \mathrm{~K}$ atoms are chosen as $\left|2^{2} P_{1 / 2}, \frac{1}{2}, \frac{1}{2}\right\rangle$ and $\left|4^{2} P_{1 / 2}, \frac{9}{2}, \frac{1}{2}\right\rangle$, respectively. So, the polarizations of the two Raman transition lasers are $\sigma^{+}$ and $\sigma^{-}$.

Through adjusting the Raman laser parameters appropriately, a magnetic $\pi$-flux lattice $[15,16,20]$ illustrated in Fig. 1(a) can be simulated and we will show this in the following. The tight-binding Hamiltonian of the lattice system takes the form

$$
H_{0}=-\sum_{\langle i, j\rangle}\left(t_{i j} \hat{a}_{i}^{\dagger} \hat{b}_{j}+\text { H.c. }\right)+\Delta \sum_{i}\left(\hat{a}_{i}^{\dagger} \hat{a}_{i}-\hat{b}_{i}^{\dagger} \hat{b}_{i}\right),
$$

where $\hat{a}_{i}^{\dagger}\left(\hat{b}_{i}^{\dagger}\right)$ is the creation operator for the internal state $|A\rangle(|B\rangle)$ at lattice site $i$ belonging to the sublattice $A(B)$, $\langle i, j\rangle$ denotes the nearest-neighbor hopping with the hopping parameter $t_{i j}=-\int w_{A}^{*}\left(\mathbf{r}-\mathbf{r}_{i}\right) \Omega_{\mathrm{eff}} w_{B}\left(\mathbf{r}-\mathbf{r}_{j}\right) d^{2} \mathbf{r}$ and $\Omega_{\mathrm{eff}}=$ $\Omega_{A}^{*} \Omega_{B} e^{i\left(\mathbf{k}_{B}-\mathbf{k}_{A}\right) \cdot \mathbf{r}}$, the spatial coordinate $\mathbf{r}=\{x, y\}$ and $w_{A, B}$ being the Wannier functions of the lowest Bloch band, and $2 \Delta$ is the tunable onsite energy imbalance between the two sublattices. For proper laser beams, we can assume $t_{i j}=$ $t_{0} e^{i A_{i j}}$, where $t_{0}>0$ is the hopping magnitude controlled by $\Omega_{A, B}$ and the overlap integral between the Wannier functions associated with each sublattice, and $A_{i j}$ is the phase induced by the wave vectors $\mathbf{k}_{A, B}$ in the LAT process $[15,16]$. For the staggered $\pi$-flux lattice shown in Fig. 1(a), we have $\sum_{\circlearrowleft} A_{i j}= \pm \pi$ for each plaquette. There are many approaches to generate the lattice with phase $A_{i j}$. A practical method with the hopping parameters $t_{x}=t_{0}$ and $t_{y}=-i t_{0}$ from sublattice $B$ to sublattice $A$, as shown in Fig. 1(c), can be found in Ref. [16]. Similar LAT schemes have been experimentally realized with bosonic atoms [20-22].

By using the Fourier transformation $\hat{a}_{j}=\frac{1}{\sqrt{N}} \sum_{\mathbf{k}} e^{i \mathbf{k} \cdot \mathbf{r}_{j}} \hat{a}_{\mathbf{k}}$ and $\hat{b}_{j}=\frac{1}{\sqrt{N}} \sum_{\mathbf{k}} e^{i \mathbf{k} \cdot \mathbf{r}_{j}} \hat{b}_{\mathbf{k}}$ on the Hamiltonian in Eq. (1) with $t_{x}=t_{0}$ and $t_{y}=-i t_{0}$, we can obtain the corresponding Hamiltonian in the momentum space as

$$
H_{\mathbf{k} 0}=\sum_{\mathbf{k}}\left(\hat{a}_{\mathbf{k}}^{\dagger}, \hat{b}_{\mathbf{k}}^{\dagger}\right)\left(\begin{array}{cc}
\Delta & f_{\mathbf{k}} \\
f_{\mathbf{k}}^{*} & -\Delta
\end{array}\right)\left(\begin{array}{l}
\hat{a}_{\mathbf{k}} \\
\hat{b}_{\mathbf{k}}
\end{array}\right),
$$

where $f_{\mathbf{k}}=-2 t_{0}\left[\cos \left(k_{x} a\right)-i \cos \left(k_{y} a\right)\right]$ with $a$ being the lattice spacing. Thus, the energy spectrum is given by $E(\mathbf{k})=$ $\pm \sqrt{\left|f_{\mathbf{k}}\right|^{2}+\Delta^{2}}$, which exhibits two inequivalent Dirac points at $K_{ \pm}= \pm \frac{\pi}{2 a}(1,1)$ with an energy gap $2 \Delta$. By substitution of $\mathbf{k} \rightarrow K_{ \pm}+\mathbf{q}$, the dynamics around the Dirac points $K_{ \pm}$(i.e., $|\mathbf{q}| a \ll 1)$ is then governed by the effective Dirac Hamiltonian $[1,9]$

$$
H_{\eta}=\eta \hbar v_{F}\left(\sigma_{x} q_{x}+\sigma_{y} q_{y}\right)+\Delta \sigma_{z},
$$

where $\eta= \pm$ represent different valleys $K_{ \pm}, v_{F}=2 t_{0} a / \hbar$ is the effective Fermi velocity, and $\sigma_{i}$ are the Pauli matrices with $i=\{x, y, z\}$. This low-energy effective Dirac Hamiltonian is similar to the unstrained nature graphene and the sublattice degree of freedom here plays the role of the spin degree of freedom.

Note that a similar Dirac Hamiltonian has been proposed in state-independent square OLs with other methods for light-induced gauge potentials [24], especially the related quantum anomalous Hall phase was also investigated in a square checkerboard lattice [25]. Although the Dirac-type equation and gauge fields have been studied in Refs. [24,25], none of them explored the valley-dependent gauge fields for cold-atomic systems. These gauge fields have been first proposed and then experimentally realized in natural and artificial graphene [5,11,13]. However, it is unclear how to create them on other lattice geometries instead of the honeycomb lattice [6]. In the following, we will propose two approaches to realize the valley-dependent gauge fields for cold atoms trapped in the square optical superlattices by modulating the hopping parameters.

To realize valley-dependent gauge fields based on the LAT method, we assume that the hopping amplitudes along the $\hat{x}$ and $\hat{y}$ axes in the previous $\pi$-flux system are further modulated by $\delta t_{x}$ and $\delta t_{y}$, respectively. The perturbation Hamiltonian for such modulation is written as

$$
\delta H=-\sum_{\langle i, j\rangle_{x}} \delta t_{x} \hat{a}_{i}^{\dagger} \hat{b}_{j}-\sum_{\langle i, j\rangle_{y}} \delta t_{y} \hat{a}_{i}^{\dagger} \hat{b}_{j}+\text { H.c. }
$$

This modulation is done by using two Raman laser beams with detuning $\Delta_{d}$, which give rise to the additional couplings being shown in Figs. 2(a) and 2(b). The Rabi frequencies of the two lasers are $\Omega_{1}(\mathbf{r}) e^{i\left(\mathbf{k}_{1} \cdot \mathbf{r}+\phi_{1}\right)}$ and $\Omega_{2}(\mathbf{r}) e^{i\left(\mathbf{k}_{2} \cdot \mathbf{r}+\phi_{2}\right)}$, where $\mathbf{k}_{l}\left(\phi_{l}\right)$ with $l=\{1,2\}$ are the wave vectors (phases) of the laser beams. Defining $\theta=\phi_{2}-\phi_{1}$ and $\mathbf{k}_{2}-\mathbf{k}_{1}=k_{x} \hat{x}+$ $k_{y} \hat{y}$, we have $\delta t_{x}=\int w_{A}^{*}\left(x_{j}, y_{j}\right) \delta \Omega e^{i\left(k_{x} x+\theta\right)} w_{B}\left(x_{j \pm 1}, y_{j}\right) d x d y$ 

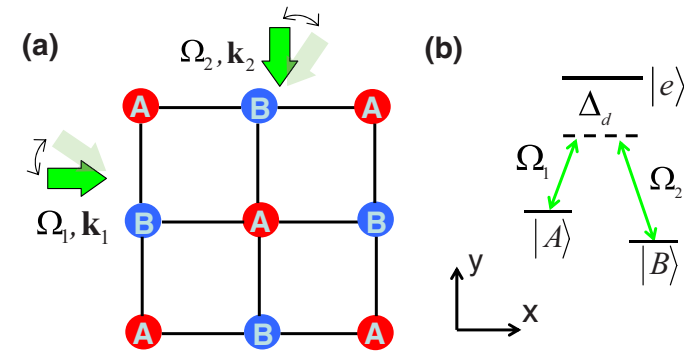

(c)

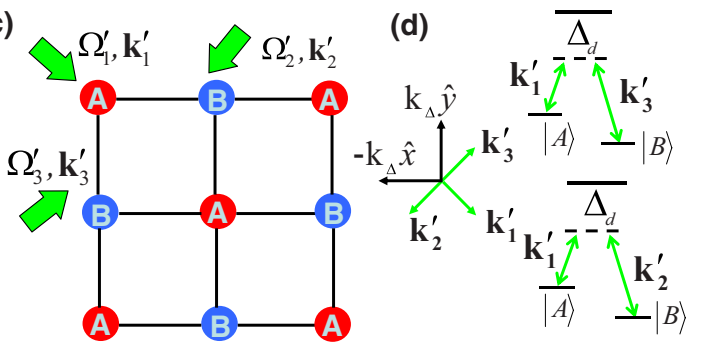

FIG. 2. (Color online) Two schemes (a), (b) and (c), (d) for generating tunable valley-dependent gauge potentials. (a) Two additional Raman beams for modulating the atomic hopping with adjustable wave vectors. (b) Sketch of the additional laser coupling between the states $|A\rangle$ and $|B\rangle$ with large detuning $\Delta_{d}$ from the state $|e\rangle$. (c) Three additional Raman beams for modulating the atomic hopping with fixed wave vectors. (d) Sketch of the additional coupling with the three lasers and the effective wave numbers $\mathbf{k}_{2}^{\prime}-\mathbf{k}_{1}^{\prime}=-k_{\Delta} \hat{x}$ and $\mathbf{k}_{3}^{\prime}-\mathbf{k}_{1}^{\prime}=k_{\Delta} \hat{y}$.

and $\delta t_{y}=\int w_{A}^{*}\left(x_{j}, y_{j}\right) \delta \Omega e^{i\left(k_{y} y+\theta\right)} w_{B}\left(x_{j}, y_{j, j \pm 1}\right) d x d y$ for the LAT $[15,16]$, where $\delta \Omega \equiv \Omega_{1} \Omega_{2} / \Delta_{d}$ and $w_{\alpha}\left(x_{j}, y_{j}\right)$ is the Wannier function on the $\alpha(=A, B)$ lattice with lattice position $\left(x_{j}, y_{j}\right)$. Generally, the modulation of the hopping parameters can be rewritten as

$$
\delta t_{x}=\delta t_{0}^{x} e^{i \varphi_{x}}, \quad \delta t_{y}=\delta t_{0}^{y} e^{i \varphi_{y}} .
$$

Here, $\delta t_{0}^{x, y}$ are the magnitudes determined by the overlap integral with respect to $\delta \Omega(\mathbf{r})$ and the Wannier functions, and $\varphi_{x, y}$ are the phases determined by $k_{x, y}$ and $\theta[15,16,20-22]$, thus the hopping modulation can be easily tuned by adjusting the parameters of the Raman laser beams. To preserve the staggered $\pi$-flux lattice and keep the previous Dirac Hamiltonian around the Dirac points, the additional flux in each plaquette has to be tuned as $2\left(\varphi_{x}+\varphi_{y}\right)=2 N \pi$ with $N=\{0, \pm 1, \pm 2, \ldots\}$. This can be achieved in experiments by appropriately adjusting the parameters $k_{x}, k_{y}$, and $\theta$ [20-22]. For example, one may set $k_{x}=k_{y}=\pi /(2 a)$ and $\theta=$ 0 , which yields $\varphi_{x}=\varphi_{y}=\pi / 2$ and satisfies the previous flux condition. We also require $\left|\delta t_{0}^{x}\right|,\left|\delta t_{0}^{y}\right| \ll t_{0}$ as a perturbation term, which can be satisfied by setting $|\delta \Omega| \ll\left|\Omega_{\text {eff }}\right|$. For example, one may tune the ratio between $\delta t_{0}^{x}\left(\delta t_{0}^{y}\right)$ and $t_{0}$ through adjusting the detuning $\Delta_{d}$.

With the previous Fourier transformation, the perturbation Hamiltonian in the momentum space is given by

$$
\delta H_{\mathbf{k}}=\sum_{\mathbf{k}} \delta f_{\mathbf{k}} \hat{a}_{\mathbf{k}}^{\dagger} \hat{b}_{\mathbf{k}}+\text { H.c. }
$$

where $\delta f_{\mathbf{k}}=-2\left[\delta t_{0}^{x} \cos \left(k_{x} a+\varphi_{x}\right)-i \delta t_{0}^{y} \cos \left(k_{y} a+\varphi_{y}\right)\right]$. If the modulation of the atomic hopping $\delta t_{0}^{x}$ and $\delta t_{0}^{y}$ is smooth over the lattice spacing scale, there is no Fourier component with $K_{+}-K_{-}$, and thus the two Dirac valleys are decoupled by the perturbation $[1,6]$. Within this smooth perturbation, the total Hamiltonian in the momentum space $H_{\mathbf{k}}=H_{\mathbf{k} 0}+\delta H_{\mathbf{k}}$ at the vicinity of the two Dirac points can be replaced by a low-energy effective spinor Hamiltonian [6]

$$
\hat{\mathcal{H}}_{\mathrm{eff}}^{\eta}=\eta v_{F} \boldsymbol{\sigma} \cdot(\hat{\boldsymbol{p}}+\mathcal{A})+\Delta \sigma_{z},
$$

where $\boldsymbol{\sigma}=\left(\sigma_{x}, \sigma_{y}\right), \hat{\boldsymbol{p}}=\left(\hat{p}_{x}, \hat{p}_{y}\right)$ is the momentum operator, and $\mathcal{A}=\left(\mathcal{A}_{x}, \mathcal{A}_{y}\right)$ with $\mathcal{A}_{x}=\frac{2}{\hbar v_{F}} \operatorname{Re}\left[\delta f_{\mathbf{k}=\mathbf{K}_{+}}\right]$and $\mathcal{A}_{y}=$ $\frac{2}{\hbar v_{F}} \operatorname{Im}\left[\delta f_{\mathbf{k}=\mathbf{K}_{+}}\right]$. Here, we expand the perturbation up to the first order of $\delta t_{0}^{x} / t_{0}$ and $\delta t_{0}^{y} / t_{0}$. We obtain $\mathcal{A}_{x}$ and $\mathcal{A}_{y}$ as

$$
\begin{aligned}
& \mathcal{A}_{x}(\mathbf{r})=\frac{2}{\hbar v_{F}} \cos \left(\varphi_{x}+\frac{\pi}{2}\right) \delta t_{0}^{x}(\mathbf{r}), \\
& \mathcal{A}_{y}(\mathbf{r})=\frac{2}{\hbar v_{F}} \cos \left(\varphi_{y}+\frac{\pi}{2}\right) \delta t_{0}^{y}(\mathbf{r}) .
\end{aligned}
$$

The Hamiltonian (7) describes the dynamics of Dirac fermions in the presence of valley-dependent gauge potentials [6]. It is obvious that $\mathcal{A}_{x}$ and $\mathcal{A}_{y}$ play the role of gauge potentials with opposite sign $\eta$ in different Dirac valleys. Here, $\mathcal{A}(\mathbf{r})$ is tunable through adjusting the parameters of Raman laser beams. For example, we can change the spatial configuration of the Rabi frequencies $\Omega_{l}(l=1,2)$ to control the position dependence of $\delta t_{0}^{x}$ and $\delta t_{0}^{y}$, and adjust the wave vectors $\mathbf{k}_{l}$ to tune $\varphi_{x}$ and $\varphi_{y}$, as demonstrated in experiments [20-22] and shown in Fig. 2(a). In this way, however, we may be unable to independently tune the spatial distributions of $\mathcal{A}_{x}$ and $\mathcal{A}_{y}$ since both of them depend on $\Omega_{1}(\mathbf{r})$ and $\Omega_{2}(\mathbf{r})$.

To enhance the tunability of the valley-dependent gauge potentials in this system, we can use three Raman beams [26] instead of two, as shown in Fig. 2(c). The Rabi frequencies of three lasers are denoted by $\Omega_{1}^{\prime}(\mathbf{r}) e^{i \mathbf{k}_{1}^{\prime} \cdot \mathbf{r}}, \Omega_{2}^{\prime}(\mathbf{r}) e^{i\left(\mathbf{k}_{2}^{\prime} \cdot \mathbf{r}+\theta^{\prime}\right)}$, and $\Omega_{3}^{\prime}(\mathbf{r}) e^{i\left(\mathbf{k}_{3}^{\prime} \cdot \mathbf{r}+\theta^{\prime}\right)}$, where $\mathbf{k}_{m}^{\prime}$ are the wave vectors with $m=$ $\{1,2,3\}$ and $\theta^{\prime}$ the relative phase. We assume the directions of the lasers are fixed as those in Fig. 2(d), with $\mathbf{k}_{2}^{\prime}-\mathbf{k}_{1}^{\prime}=-k_{\Delta} \hat{x}$ and $\mathbf{k}_{3}^{\prime}-\mathbf{k}_{1}^{\prime}=k_{\Delta} \hat{y}$. Then, the effective hopping-modulation parameters along the $\hat{x}$ and $\hat{y}$ axes $\left(\delta t_{x}\right.$ and $\left.\delta t_{y}\right)$ are replaced by $\delta t_{x}^{\prime}=\int w_{A}^{*}\left(x_{j}, y_{j}\right) \delta \Omega_{x} e^{i\left(-k_{\Delta} x+\theta^{\prime}\right)} w_{B}\left(x_{j \pm 1}, y_{j}\right) d x d y$ and $\delta t_{y}^{\prime}=\int w_{A}^{*}\left(x_{j}, y_{j}\right) \delta \Omega_{y} e^{i\left(k_{\Delta} y+\theta^{\prime}\right)} w_{B}\left(x_{j}, y_{j, j \pm 1}\right) d x d y$, with $\delta \Omega_{x} \equiv \Omega_{1}^{\prime} \Omega_{2}^{\prime} / \Delta_{d}$ and $\delta \Omega_{y} \equiv \Omega_{1}^{\prime} \Omega_{3}^{\prime} / \Delta_{d}$. In this case, we can independently tune the spatial distributions of $\mathcal{A}_{x}$ and $\mathcal{A}_{y}$ through adjusting $\Omega_{2}^{\prime}$ and $\Omega_{3}^{\prime}$, respectively.

When the hopping modulations $\delta t_{0}^{x}$ and (or) $\delta t_{0}^{y}$ are time dependent, the gauge potential in Eq. (8) also becomes time dependent, as the one in nature graphene under timedependent strains [27,28]. Therefore, in general cases, we have $\mathcal{A}(\mathbf{r}, t)$, which is associated with a valley-dependent effective electromagnetic field $\left\{\mathbf{E}_{\eta}, \mathbf{B}_{\eta}\right\}$ given by

$$
\mathbf{E}_{\eta}=-\eta \partial \mathcal{A} / \partial t, \quad \mathbf{B}_{\eta}=\eta \nabla \times \mathcal{A} .
$$

The time-dependent modulations in this system can be easily realized by varying the Rabi frequencies (i.e., their laser intensities) of the Raman beams or the detuning with time. Interestingly, a time-dependent but valley-independent vector potential associated with an effective electric field for neutral atoms was created by tuning the detuning in the laser-atom coupling with time [29]. In contrast, we have proposed a 
feasible scheme to realize the tunable valley-dependent gauge fields (including electric field) in a square optical superlattice. We note that our scheme can be extended to simulate the nonAbelian $S U(2)$ valley-dependent gauge fields by introducing density waves or double layers with proper additional optical potentials, similar to the proposals in molecular graphene [30] and bilayer graphene [31].

We now present some potential applications with the tunable valley-dependent gauge fields in this system. First, we consider QVHE with valley-Landau levels (VLLs) [4,5] which requires a uniform valley-dependent magnetic field $\mathbf{B}_{\eta}=\eta B_{0} \hat{z} \quad\left(B_{0}>0\right)$, corresponding to the Landau gauge $\mathcal{A}=\left(-B_{0} y, 0\right)$. Realization of this gauge potential requires carefully designing the laser configurations [32] and would-be challenge as the case in graphene [4]. The eigenstates of $\hat{\mathcal{H}}_{\text {eff }}^{\eta}$ with $\Delta=0$ then fall into the quantized VLLs at energies $E_{n}=\operatorname{sgn}(n) \sqrt{2 \hbar v_{F}^{2} B_{0}|n|}$ [1]. Because $\mathbf{B}_{\eta}$ has opposite signs for carriers in valleys $K_{+}$and $K_{-}$, the chiral edge states protected by the bulk gap $\left|E_{n+1}-E_{n}\right|$ at the boundary are counterpropagating with different valleys. This is in contrast to the copropagating ones for a real magnetic field, and is reminiscent of topological insulators and therefore is called QVHE characterized by zero-charge Chern number and a nonzero valley Chern number [33]. Interestingly, the $n=0$ VLL wave functions in both valleys are localized entirely on the sublattice $B$ [7]. In our system, atoms in the sublattice $B$ have the internal state $|B\rangle$, which is convenient for spin-resolved observation. Second, this system with tunable valley-dependent electromagnetic fields provides an ideal platform to explore atomic devices based on valley-dependent gauge fields [34]. When the system is metallic, the valley-dependent electric field can be used to drive valley currents and further to design atomic valley filters $[34,35]$. We can also simulate valley Hall effects by the valley-dependent magnetic field. In this case, the pseudomagnetic field can be nonuniform and even zero as $\mathcal{A}$ is a nonzero constant, which is much easier to be achieved by selecting the laser configurations [32]. Note that atomic spin Hall effects [36] have been observed in a very recent experiment [37]. Similarly, by subjecting the system to a valley-dependent electric field, we may produce an atomic topological edge current related to the valley degree of freedom [28].

Finally, we briefly discuss the feasibility of our proposal with the practical experimental parameters. Let us consider ${ }^{40} \mathrm{~K}$ atoms with the typical lattice spacing $a \simeq 400 \mathrm{~nm}$ and lattice depth $V_{0} \simeq 22 E_{r}$ [38], where $E_{r}$ is the recoil energy. With the typical choice $E_{r} / \hbar \simeq 8 \mathrm{kHz}$, numerical simulations in Ref. [38] indicate that the band gap between the two lowest Bloch bands $\Delta E_{\text {gap }} \simeq 8 E_{r}$ and the natural (nextnearest-neighbor) hopping within sublattices $t_{N} \lesssim 10^{-3} E_{r}$. The nearest-neighbor hopping $\left|t_{i j}\right| \equiv t_{0}$ is proportional to the effective Raman intensity $\left|\Omega_{\text {eff }}\right|$, and $t_{0} \gtrsim \hbar\left|\Omega_{\text {eff }}\right| \beta$ with the overlap integral of Wannier functions between neighbor lattice sites $\beta \simeq 10^{-2}$. So, a feasible value $\left|\Omega_{\text {eff }}\right| \sim 10 E_{r} / \hbar$ would not pump the atoms into the higher Bloch bands. For typical hopping perturbation $\delta t_{0}^{x, y} \sim 0.1 t_{0} \gtrsim 10 t_{N}$, the natural hopping terms can be neglected safely. The spontaneous emission and the associated atomic heating are also negligible within several seconds in current experiments [20-22].

In summary, we have proposed an experimental scheme to simulate the tunable valley-dependent gauge fields with ultracold fermionic atoms in a square optical superlattice using the LAT method. Our scheme provides a pathway to explore the quantum valley Hall effects and atomic devices based on valley-dependent gauge fields. In view of the fact that the LAT technique has been demonstrated in similar OLs in recent experiments [20-22], it is anticipated that the present proposal will be tested in an experiment in the near future.

This work was supported by the NSFC (Grants No. 11125417, No. 11274124, and No. 11174088), the SKPBR (Grant No. 2011CB922104), NCET (Grant No. 10-0090), the PCSIRT, and the SRFGS of SCNU.
[1] A. H. C. Neto, F. Guinea, N. M. R. Peres, K. S. Novoselov, and A. K. Geim, Rev. Mod. Phys. 81, 109 (2009).

[2] A. F. Morpurgo and F. Guinea, Phys. Rev. Lett. 97, 196804 (2006).

[3] V. M. Pereira and A. H. Castro Neto, Phys. Rev. Lett. 103, 046801 (2009).

[4] F. Guinea, M. I. Katsnelson, and A. K. Geim, Nat. Phys. 6, 30 (2009); F. Guinea, A. K. Geim, M. I. Katsnelson, and K. S. Novoselov, Phys. Rev. B 81, 035408 (2010).

[5] N. Levy, S. A. Burke, K. L. Meaker, M. Panlasigui, A. Zettl, F. Guinea, A. H. C. Neto, and M. F. Crommie, Science 329, 544 (2010); D. Guo, T. Kondo, T. Mmachida, K. Iwatake, S. Okada, and J. Nnakamura, Nat. Commun. 3, 1068 (2012); W. Yan, W.-Y. He, Z.-D. Chu, M. Liu, L. Meng, R.-F. Dou, Y. Zhang, Z. Liu, J.-C. Nie, and L. He, ibid. 4, 2159 (2013).

[6] For a review, see A. H. Vozmediano, M. I. Katsnelson, and F. Guinea, Phys. Rep. 496, 109 (2010).

[7] P. Ghaemi, J. Cayssol, D. N. Sheng, and A. Vishwanath, Phys. Rev. Lett. 108, 266801 (2012); D. A. Abanin and D. A. Pesin, ibid. 109, 066802 (2012); B. Roy and I. F. Herbut, Phys. Rev. B 88, 045425 (2013).
[8] For a pedagogical review, see M. Polini, F. Guinea, M. Lewenstein, H. C. Manoharan, and V. Pellegrini, Nat. Nanotechnol. 8, 625 (2013).

[9] S. L. Zhu, B. Wang, and L.-M. Duan, Phys. Rev. Lett. 98, 260402 (2007); D. W. Zhang, Z. D. Wang, and S. L. Zhu, Front. Phys. 7, 31 (2012).

[10] L. Tarruell, D. Greif, T. Uehlinger, G. Jotzu, and T. Esslinger, Nature (London) 483, 302 (2012).

[11] M. C. Rechtsman, J. M. Zeuner, A. Tünnermann, S. Nolte, M. Segev, and A. Szameit, Nat. Photonics 7, 153 (2013).

[12] M. Bellec, U. Kuhl, G. Montambaux, and F. Mortessagne, Phys. Rev. Lett. 110, 033902 (2013).

[13] K. K. Gomes, W. Mar, W. Ko, F. Guinea, and H. C. Manoharan, Nature (London) 483, 306 (2012).

[14] See the two reviews and the references therein, J. Dalibard, F. Gerbier, G. Juzeliūnas, and P. Öhberg, Rev. Mod. Phys. 83, 1523 (2011); N. Goldman, G. Juzeliūnas, P. Öhberg, and I. B. Spielman, arXiv:1308.6533.

[15] D. Jaksch and P. Zoller, New J. Phys. 5, 56 (2003); E. J. Mueller, Phys. Rev. A 70, 041603 (2004). 
[16] F. Gerbier and J. Dalibard, New J. Phys. 12, 033007 (2010); L. Mazza, A. Bermudez, N. Goldman, M. Rizzi, M. Angel Martin-Delgado, and M. Lewenstein, ibid. 14, 015007 (2012).

[17] L. B. Shao, S. L. Zhu, L. Sheng, D. Y. Xing, and Z. D. Wang, Phys. Rev. Lett. 101, 246810 (2008).

[18] E. Alba, X. Fernandez-Gonzalvo, J. Mur-Petit, J. K. Pachos, and J. J. Garcia-Ripoll, Phys. Rev. Lett. 107, 235301 (2011).

[19] E. Alba, X. Fernandez-Gonzalvo, J. Mur-Petit, J. J. GarciaRipoll, and J. K. Pachos, Ann. Phys. (NY) 328, 64 (2013).

[20] M. Aidelsburger, M. Atala, S. Nascimbène, S. Trotzky, Y.-A. Chen, and I. Bloch, Phys. Rev. Lett. 107, 255301 (2011).

[21] M. Aidelsburger, M. Atala, M. Lohse, J. T. Barreiro, B. Paredes, and I. Bloch, Phys. Rev. Lett. 111, 185301 (2013).

[22] H. Miyake, G. A. Siviloglou, C. J. Kennedy, W. C. Burton, and W. Ketterle, Phys. Rev. Lett. 111, 185302 (2013).

[23] O. Mandel, M. Greiner, A. Widera, T. Rom, T. W. Hansch, and I. Bloch, Phys. Rev. Lett. 91, 010407 (2003); P. J. Lee, M. Anderlini, B. L. Brown, J. Sebby-Strabley, W. D. Phillips, and J. V. Porto, ibid. 99, 020402 (2007).

[24] L.-K. Lim, C. M. Smith, and A. Hemmerich, Phys. Rev. Lett. 100, 130402 (2008); J. M. Hou, W. X. Yang, and X.-J. Liu, Phys. Rev. A 79, 043621 (2009); N. Goldman, A. Kubasiak, A. Bermudez, P. Gaspard, M. Lewenstein, and M. A. MartinDelgado, Phys. Rev. Lett. 103, 035301 (2009); N. Goldman, I. Satija, P. Nikolic, A. Bermudez, M. A. Martin-Delgado, M. Lewenstein, and I. B. Spielman, ibid. 105, 255302 (2010); X. J. Liu, X. Liu, C. Wu, and J. Sinova, Phys. Rev. A 81, 033622 (2010); F. Mei, S.-L. Zhu, X.-L. Feng, Z.-M. Zhang, and C. H. Oh, ibid. 84, 023622 (2011); N. R. Cooper, Phys. Rev. Lett. 106, 175301 (2011).

[25] N. Goldman, F. Gerbier, and M. Lewenstein, J. Phys. B: At., Mol. Opt. Phys. 46, 134010 (2013).

[26] Y.-H. Chan and L.-M. Duan, New J. Phys. 14, 113039 (2012).
[27] F. von Oppen, F. Guinea, and E. Mariani, Phys. Rev. B 80, 075420 (2009).

[28] A. Vaezi, N. Abedpour, R. Asgari, A. Cortijo, and M. A. H. Vozmediano, Phys. Rev. B 88, 125406 (2013).

[29] Y.-J. Lin, R. L. Compton, K. Jiménez-García, W. D. Phillips, J. V. Porto, and I. B. Spielman, Nat. Phys. 7, 531 (2011).

[30] S. Gopalakrishnan, P. Ghaemi, and S. Ryu, Phys. Rev. B 86, 081403 (2012); F. de Juan, ibid. 87, 125419 (2013).

[31] P. San-Jose, J. González, and F. Guinea, Phys. Rev. Lett. 108, 216802 (2012).

[32] The magnitude of the hopping perturbation is approximately proportional to the effecive intensity of the additional Raman beams. Therefore, to induce the uniform pseudomagnetic field, one may require $|\delta \Omega(x, y)| \propto y$ along the $\hat{x}$ axis. For the running-wave Raman lasers, the synthetic gauge potential is position independent and then $\mathbf{B}_{\eta}=0$; for the standing-wave or Gaussian-type lasers, $\mathbf{B}_{\eta}$ is position dependent but nonuniform.

[33] W. Yao, S. A. Yang, and Q. Niu, Phys. Rev. Lett. 102, 096801 (2009); F. Zhang, J. Jung, G. A. Fiete, Q. Niu, and A. H. MacDonald, ibid. 106, 156801 (2011).

[34] A. Rycerz, J. Tworzydlo, and C. W. J. Beenakker, Nat. Phys. 3, 172 (2007); D. Xiao, W. Yao, and Q. Niu, Phys. Rev. Lett. 99, 236809 (2007).

[35] G.-B. Zhu, Q. Sun, Y.-Y. Zhang, K. S. Chan, W.-M. Liu, and A.-C. Ji, Phys. Rev. A 88, 023608 (2013).

[36] S. L. Zhu, H. Fu, C. J. Wu, S.-C. Zhang, and L.-M. Duan, Phys. Rev. Lett. 97, 240401 (2006); X. J. Liu, X. Liu, L. C. Kwek, and C. H. Oh, ibid. 98, 026602 (2007).

[37] M. C. Beeler, R. A. Williams, K. Jiménez-García, L. J. LeBlanc, A. R. Perry, and I. B. Spielman, Nature (London) 483, 201 (2013).

[38] J. K. Pachos, E. Alba, V. Lahtinen, and J. J. Garcia-Ripoll, Phys. Rev. A 88, 013622 (2013). 